\title{
Electromagnetic Waves Propagation in Unconventional Doubly Clad Optical Waveguide
}

\author{
Shishu Pal Singh' ${ }^{1}$ Anshika Singh ${ }^{2}$ \\ Department of Physics, Ramkishun Singh Degree College, Siddiqpur, Jaunpur (U.P) ${ }^{1}$. \\ M.Sc.in Physics, Department of Physics, J.S. University, Shikohabad, Firozabad (U.P)².
}

\begin{abstract}
The modal dispersion and cutoff conditions for a W-type plasma loaded circular waveguide are presented. The proposed waveguide has three parts namely the core with higher index, the inner cladding with plasma and outer cladding with air. The dispersion equation is obtained by solving the Maxwell's equation and matching the field at various interfaces. The graphs for modal characteristics are presented. It is noted as the width of plasma layer increases, the cutoff frequency also increases considerably in all considered case. This analysis shows that the introduction of plasma in waveguide provides additional parameters for controlling the propagation property.
\end{abstract}

\section{INTRODUCTION.}

Optical fiber and optical waveguides have been integral part of the modern communication systems [1-13]. The development of high quality optical fiber technology, the optical communication industry has witnessed an enormous growth in the last four decades. Fiber optics is now taken for granted in view of wide-ranging application as the most suitable singular medium for transmission of voice; video and data signals. Indeed, optical fibers have now penetrated virtually all segments of telecommunication networks. The most popular waveguide for optical communication is the standard optical fiber having circular cross-section core cross-section. However; planar; rectangular; triangular; super elliptical and hypocycloidal waveguides are also widely known for their use in integrated optical devices, laser beam technology and optical fiber based sensors [6-10]. The first communication expert to direct serious attention to glass fibers for long distance was Kao, then at standard telecommunication laboratories in England. At that time [1968] typical fiber losses were above 1000db/Km; but Kao suggested that purer materials should permit much lower losses. In1970, Kapron, Keck and Maurer announced the achievement of losses under $20 \mathrm{db} / \mathrm{Km}$ in single mode fibers hundreds of meter long. Moreover the doubly clad optical waveguides is a novel type of optical waveguides having various advantages on the single clad fiber [14-15]. It consists of the three portions; core has the highest refractive index $\mathrm{n}_{1}$; the intermediate layer (inner cladding) has the lowest index $\mathrm{n}_{2}$ and outermost part (outer cladding) has the index $n_{3}$. All three portions are assumed to be project insulators with the free space permeability $\mu_{0}$ and also assumed that outer cladding extends to infinity. The radius of the core is denoted by ' $a$ ' and thickness of the inner cladding is ' $b$ '. There is several method of making a W-type fiber. This waveguides differ from the standard circular step-index fibers in respect of bounded condition of light wave. The Chemical Vapor-Deposition Technique [CVDT] is one the promising methods [16-17]. In this paper we will explore some of the fundamental properties of a doubly clad optical waveguides with especial reference to their use in optical communications system. Here we are study the propagation properties, modal characteristics equations, modal cut off conditions; dispersion curves and other relevant properties of a doubly clad optical waveguides by using analytical methods of studied waveguides in view of theoretical under standing. Hence the present paper reports the finding of a theoretical study of modal properties of a double clad optical waveguides. Although the proposed waveguide presents a complicated mathematical problem and therefore; we do not attempt to describe in details the various complex mode and corresponding modal fields which will be generated in such a system; but restrict our selves to the estimation of the propagation constant only.

\section{THEORETICAL BACKGROUND:}

Here we will use a simple matrix method to compute the modal characteristics of a doubly clad waveguide. The basic idea is to replace the boundary condition by a matrix equation. The cross-sectional view of doubly clad waveguide is shown in Fig.1. The core has the largest refractive index of the three, and the outer cladding index is the next largest. Thereby we have suitably designed alternating claddings of low and high refractive indices. The index profile is shown in Fig. 2.

We choose the cylindrical coordinate system for proposed geometry and assume that electromagnetic wave propagates along the Z-axis. Using these coordinates and Maxwell equations we can obtain the expressions for the field $\mathrm{E}$ and $\mathrm{H}$ in terms of 


\section{DOI: $10.17148 /$ IJIREEICE.2022.10114}

the new coordinates. The details of this procedure are given in a classical paper [1-2]. The axial field components can be written as

$$
\begin{aligned}
& \left.\begin{array}{l}
E_{z}=A J_{n}(u r) \cos n \theta \\
H_{z}=B J_{n}(u r) \sin n \theta
\end{array}\right\} \quad \text { for core region } \\
& \left.\begin{array}{l}
E_{z}=C I_{n}\left(w_{1} r\right)+D K_{n}\left(w_{1} r\right) \cos n \theta \\
H_{z}=F I_{n}\left(w_{1} r\right)+G K_{n}\left(w_{1} r\right) \sin n \theta
\end{array}\right\} \quad \text { for inner cladding region } \\
& \left.\begin{array}{l}
E_{z}=L K_{n}\left(w_{2} r\right) \cos n \theta \\
H_{z}=M K_{n}\left(w_{2} r\right) \sin n \theta
\end{array}\right\} \quad \text { outer cladding region }
\end{aligned}
$$

where $u^{2}=\omega^{2} n_{1}^{2} \mu_{1}-\beta^{2}, w_{1}{ }^{2}=\beta^{2}-\omega^{2} \mu_{2} n_{2}^{2}$ and $w_{2}{ }^{2}=\beta^{2}-\omega^{2} \mu_{3} n_{3}^{2}$

Also A, B, C, D, F,G,L and M are unknown constants and $n_{1}, n_{2}$, and $n_{3}$ are the permittivity of the core, inner and outer cladding region respectively . Here $\mu_{1}=\mu_{2}=\mu_{3}=\mu_{0}$ is the permeability of the medium. $J_{n}$ is the Bessel function for the guiding region $I_{n}$ and $K_{n}$ are the modified Bessel function for the inner and outer cladding regions respectively.

Here $\beta$ is the axial component of propagation vector, $\omega$ is the wave frequency, $\mu_{0}$ is the permeability of non-magnetic medium. The boundary conditions can be written as

$$
\begin{aligned}
& \left.E_{\xi 1}\right|_{r}=\left.E_{\xi 2}\right|_{r} \\
& \left.\frac{\partial E_{\xi 1}}{\partial \xi}\right|_{r}=\left.\frac{\partial E_{\xi 2}}{\partial \xi}\right|_{r}
\end{aligned}
$$

Thus we get a set of equations having four unknown constants. The nontrivial solution will exist only when the determinant formed by the coefficients of the unknown constants is equal to zero. Hence we have

$$
\left|\begin{array}{cccc}
J_{n}(u r) & -I_{n}\left(w_{1} r\right) & -K_{n}\left(w_{1} r\right) & 0 \\
u J_{n}^{\prime}(u r) & -w_{1} I_{n}^{\prime}\left(w_{1} r\right) & -w_{1} K_{n}^{\prime}\left(w_{1} r\right) & 0 \\
0 & I_{n}\left(w_{1} r\right) & K_{n}\left(w_{1} r\right) & -K_{n}\left(w_{2} r\right) \\
0 & w_{1} I_{n}^{\prime}\left(w_{1} r\right) & w_{1} K_{n}^{\prime}\left(w_{1} r\right) & -w_{2} K_{n}^{\prime}\left(w_{2} r\right)
\end{array}\right|=0
$$

The dimensionless V-parameter is introduced to incorporate the parameters $n_{1}, n_{2}, n_{3}, a$, and $k_{0}$ which may possibly have an effect on the propagation.

$V=k_{0} a\left(n_{1}^{2}-n_{2}^{2}\right)^{\frac{1}{2}}$

where $k_{0}$ is vacuum wavenumber. We define the usual normalized propagation parameter

$b^{\prime}=\frac{\beta^{2}-k_{0}^{2} n_{3}^{2}}{k_{0}^{2}\left(n_{1}^{2}-n_{3}^{2}\right)} \quad$ (weakly guidance case) 
International Journal of Innovative Research in Electrical, Electronics, Instrumentation and Control Engineering

Impact Factor 7.047 Vol. 10, Issue 1, January 2022

DOI: 10.17148/IJIREEICE.2022.10114

\section{NUMERICAL COMPUTATIONS, RESULTS AND DISCUSSION:}

The characteristic equation (4) contains all of the information that we can obtain from our modal analysis and it gives the central results of this investigation. We now proceed to some numerical computation in order to have the modal dispersion curves for the proposed waveguide. It is convenient to plot the normalized propagation constant $b^{\prime}=\frac{\frac{\beta^{2}}{k_{0}^{2}}-n_{3}^{2}}{n_{1}^{2}-n_{3}^{2}}$ against the V-parameter defined by $V=\frac{2 \pi a}{\lambda_{0}}\left(n_{1}^{2}-n_{2}^{2}\right)^{\frac{1}{2}}$. Now we choose the refractive indices $n_{1}=1.50, n_{2}=1.20, n_{3}=1.30$ for core, inner cladding and outer cladding respectively also an operating wavelength $\lambda_{0}=1.55 \mu m$ and various values of dimensional parameter $a$ in a regular increasing order. For each value of $a$ we obtain the V-parameter and also compute the values of $\beta$ from the characteristic equation (4) by graphical method. It means that the left hand side of characteristic equation is plotted against $\beta$ for the assumed value of $a$ and the zero crossing of the graph with the $\beta$ axis are noted. These values are the solutions of the characteristic equation for the different modes. For example the lowest zero crossing value of $\beta$ corresponds to the lowest order mode. From these value of $\beta$ we can compute the values of $b^{\prime}$ and then plot the dispersion curves for the different modes. These graphs are shown in figure (3) for these modes. These dispersion curves have the expected shape, which means that the doubly clad waveguide does not cause a change in the shape of dispersion curves. The cutoff frequencies obtained from the characteristic equation (4) for these modes are at $\mathrm{V}=1.82, \mathrm{~V}=5.83$ and $\mathrm{V}=9.48$ respectively.

\section{Figure Caption :}

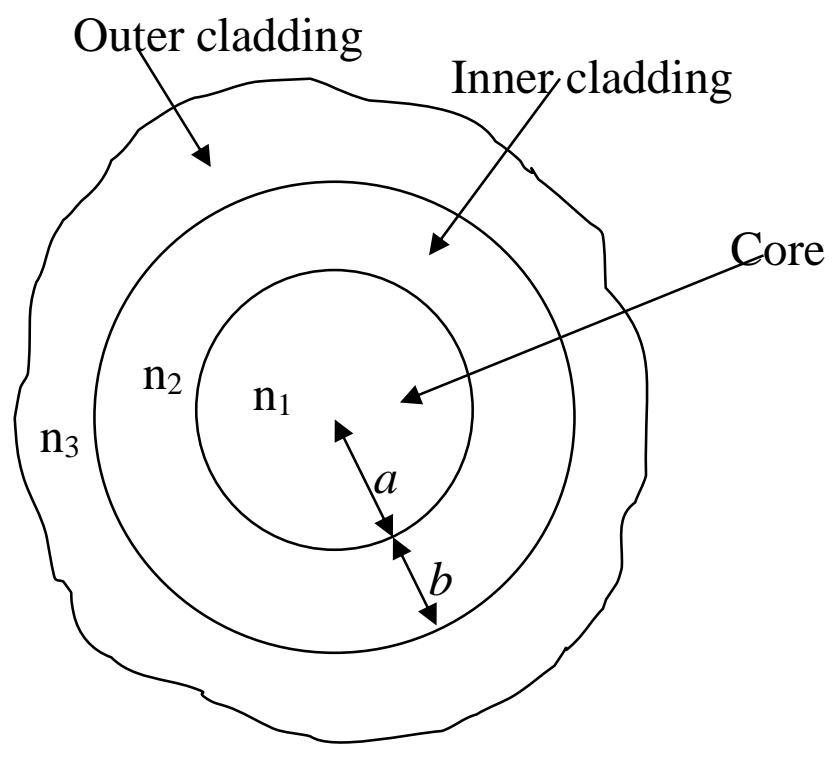

Fig. 1

Fig. 1. Geometry of the proposed doubly clad optical waveguide 
International Journal of Innovative Research in Electrical, Electronics, Instrumentation and Control Engineering

Impact Factor $7.047 \div$ Vol. 10, Issue 1, January 2022

DOI: 10.17148/IJIREEICE.2022.10114

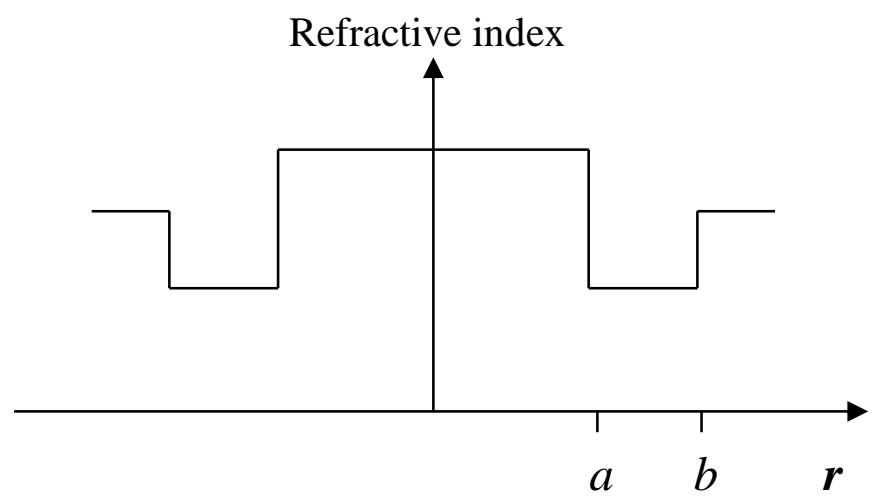

Fig. 2

Fig. 2. Index profile of a weakly guiding fiber waveguide.

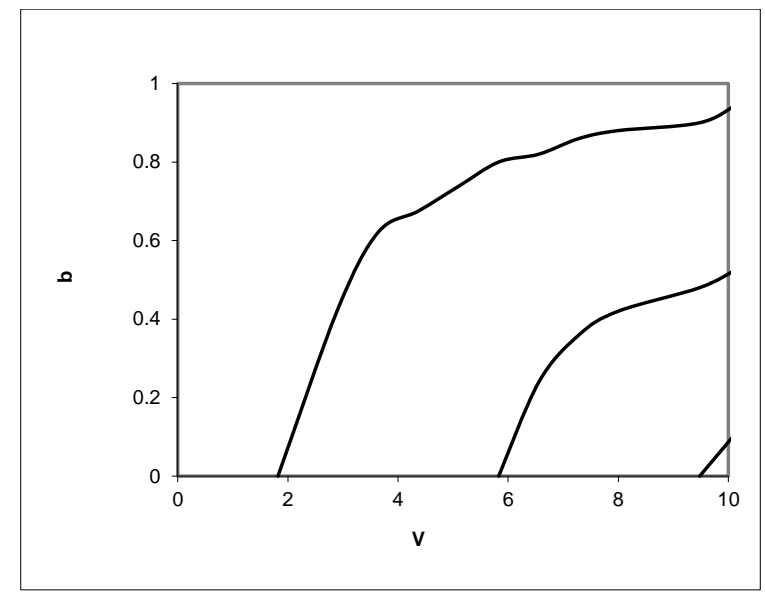

Fig. 3

Fig. 3. Dispersion curves $\left(b^{/}\right.$versus $\left.\mathrm{V}\right)$ of a few lowest modes for the proposed waveguide.

\section{REFERENCES:}

1. Snitzer, E.: Cylindrical dielectric waveguides. J. Opt. Soc. Am, 51 (1961) 491-498.

2. Snyder, A.W. and D.J. Mitchell, : Leaky rays on circular fibers. J. Opt. Soc. Am, 64 (1974) 599-607.

3. Snyder, A.W., : Understanding of monomode optical fibers. Proc. IEEE, 69 (1981) 6-13.

4. Ghatak, A and A.Sharma, : Single mode fiber characteristics. J. Inst. Electron. Telecommun. Eng., 32 (1986) 213-226.

5. Dyott R.B., : Cutoff of the first order modes in elliptical dielectric waveguide: an experimental approach. Electron Lett. 26 (1990) 1721-1723.

6. Yeh C., : Modes in weakly guiding elliptical optical fibers. Opt. Quantum Electron, 8 (1976) $43-47$.

7. Goell J.E., : A circular harmonic computer analysis of rectangular dielectric waveguides. Bell Syst. Tech. J. 48 (1969) 2133-2160.

8. Singh, V., B.Prasad and S.P.Ojha, : Modal analysis and dispersion curves of an annular lightguide with a cross-section bounded by two Piet-Hein curves. J. Electromagnetic waves and Applications 17 (2003) 1025 1036. 


\section{IJIREEICE}

ISSN (O) 2321-2004, ISSN (P) 2321-5526

International Journal of Innovative Research in Electrical, Electronics, Instrumentation and Control Engineering

Impact Factor $7.047 \approx$ Vol. 10, Issue 1, January 2022

DOI: $10.17148 / I J I R E E I C E .2022 .10114$

9. Joshi, M., V. Singh, B.Prasad and S.P.Ojha, : An analytical study of the cutoff conditions and dispersion curves of an optical fiber with a core slightly flattened on one side. Microwave Opt. Technol.Lett, 29 (2001) 136-139.

10. Singh, V., B.Prasad and S.P.Ojha, : Weak guidance modal analysis and dispersion curves of an infraredlightguide having a core cross-section with a new type of asymmetric loop boundary. Optical Fiber Technology, 6 (2000) 290-298.

11. Mishra ,V.N., V. Singh, B.Prasad and S.P.Ojha.: An analytical investigation of the dispersion characteristics of a lightguide with an annular core cross section bounded by two cardioids, Microwave Opt. Technol. Lett., 23 (1999) 221-224.

12. Stern, M.S., : Semivectorial polarized finite difference method for optical waveguides with arbitrary index profile. in IEE Proc. J. 135 (1988) 56-63.

13. Kendall, P.C., Mcllroy, P.W.A. and Stern M.S., : Spectral index method for rib waveguide analysis. Electron Lett. 25, (1989) 107-108.

14. Kawakami S. and S. Nishida, "Characteristic of a doubly clad optical fiber with a low -index inner cladding, Journal of Quantum Electronics, QE-10, 870-887, 1974.

15. Kawakami S. and S. Nishida, "Perturbation Theory of a Doubly Clad Optical Fiber with a Low-Index Inner Cladding, Journal of Quantum Electronics, QE-11, 130-138, 1975.

16. W. G. French, A. D. Pearson, G. W. Tasker, and J. B. Mac- Chesney, "Low-loss fused silica optical waveguide with borosilicate cladding," Awl. Phys. Lett., 1-01, 23, pp. 338-339, 1973.

17. J. E. MacChesney et al., "Low-loss silica core-borosilicate clad fiber optical waveguide," Appl. Phys. Lett., vol. 23, pp.340-341, 1973.

18. Lui, W.W, Xu C.L, Huang W.P., Yokoyama K. and Seke S., Full vector mode analysis with considerations of field singularities at corners of optical waveguides, J.Lightwave Technol. 17, 1509-1513, 1999.

19. Riley KF, Hobson MP, Bence ST, : Mathematical method for physics and engineering. Cambridge University Press, Cambridge, England, (1998) 274-280.

20. Singh. V., M. Joshi, B. Prasad and S.P.Ojha. : Modal dispersion characteristics and waveguide dispersion of an optical waveguide having a new unconventional core cross section, J. Electromagn. Waves and Appl. 18 (2004) 455-468.

21. Adams, M.J. : An introduction to optical waveguides. John Wiley, Chichester (1981) 233-239. 